\title{
LOS COSTOS OCULTOS DEL PRESENTISMO LABORAL EN LAS EMPRESAS MANUFACTURERAS DE LIMA: 2012
}

\author{
THE HIDDEN COSTS OF LABOR PRESENTEEISM \\ IN LIMA MANUFACTURING COMPANIES: 2012 \\ Daniel Hernán Valera LozA* \\ Unidad de Posgrado de la Facultad de Ciencias Contables \\ Universidad Nacional Mayor de San Marcos-UNMSM / Lima-Perú \\ [Recepción: Marzo de 2015 / Conformidad: Marzo de 2015]
}

\section{RESUMEN}

El presente trabajo tuvo como objetivo que mediante un diseño descriptivo correlacional, se ha determinado los costos del presentismo laboral en las empresas manufactureras de Lima, durante el año 2012. Se ha aplicado un cuestionario estructurado a 53 empresas manufactureras de Lima, encuestándose a un total de 903 trabajadores y trabajadoras, siendo el 36.7\% varones y el $63.3 \%$ mujeres. El cuestionario fue previamente validado en una muestra nacional, en más de 211 empresas, y contuvo las escalas de presentismo, ausentismo y tardanza de personal, todos con valores altos de consistencia interna y validez de constructo mediante el análisis factorial. Se encontraron como resultados que los costos del presentismo laboral representaron para Lima una pérdida significativa de 3 mil 725 millones 463 mil nuevos soles por año, un equivalente a 1,432 millones 870 mil dólares americanos por año, o al $0.78 \%$ del Producto Bruto Interno. Además, los costos del presentismo, para el sector de manufactura, representaron un alto porcentaje de los costos totales (75.2\%), en comparación de los costos por ausentismo (23.74\%) y tardanzas (1.04\%). Se ha recomendado crear modelos de gestión del desempeño que incluya dentro de sus componentes, el controly manejo del presentismo, enfatizándose la prevención.

\section{Palabras clave:}

Presentismo; costos labores; manufactura; Lima; trabajadores; ausentismo.

\begin{abstract}
The present study though the use of a descriptive correlational design, determined the costing of labor presenteeism in manufacturing companies in Lima, in 2012. A structured questionnaire was applied on 53 manufacturers of Lima, surveying a total of 903 workers, being $36.7 \%$ men and $63.3 \%$ women. The questionnaire was previously validated in a national sampling, in more than 211 companies, and held the scales of presenteeism, absenteeism and tardiness of staff, all with high levels of internal consistency and constructed validity using factor analysis. We found the result that labor costs accounted for Lima presenteeism has a significant loss of $3,725,463,000$ soles per year, equivalent to $1,432,870,000$ US dollars per year, or $0.78 \%$ of Gross Domestic Product. In addition, the costs of presenteeism, for the manufacturing sector represented a high percentage of total costs $(75.2 \%)$ compared to the costs of absenteeism $(23.74 \%)$ and tardiness (1.04\%). It is recommended to create models of performance management that includes within its components the control and management of presenteeism, emphasizing prevention.
\end{abstract}

\section{Keywords:}

Presenteeism; labor costs; manufacturing; Lima; workers; absenteeism.

\footnotetext{
* Doctorante en Ciencias Contables y Empresariales - UNMSM. Doctor en Administración por la Universidad Nacional Federico Villarreal. Email: dvaleral@usmp.pe
} 


\section{INTRODUCCIÓN}

Una forma de mejorar la competitividad de las empresas es incrementando la productividad y el valor agregado. Por ello, las estrategias clave en la gestión de recursos humanos de toda empresa se deben enfocar en el capital humano, tratando de aumentar la productividad laboral (Fernández, Avella, \& Fernández, 2003). Algunos indicadores antagónicos a estos esfuerzos son el ausentismo y la rotación de personal, los cuales traen grandes costos a las empresas. Sin embargo, hay una variable, el presentismo que puede estar acarreando costos mayores que el ausentismo y la rotación juntos.

El presentismo laboral ha sido definido de diversas formas. De acuerdo a Schultz, Chin-Yu \& Edington (2009) y Aronsson \& Gustafsson (2005) el presentismo es la disminución de la productividad de los trabajadores debido a problemas de salud o personales, y que la mayoría de las veces pasa desapercibido por las gerencias. Estimar el impacto del presentismo en la productividad laboral y determinar los costos que originan, son preocupaciones que recientemente están surgiendo en el mundo académico (Johns, 2010; García, 2011).

Diversas investigaciones a nivel mundial, han tratado de estimar los costos del presentismo. En Estados Unidos, las empresas pierden entre \$150 a \$ 250 billones de dólares anuales a causa del presentismo, los cuales se estiman son el 63\% de la totalidad de los costos empresariales (Hemp, 2004). Se afirma que los costos que afectan más a la competitividad de la empresas o la pérdida del desempeño en el trabajo, son los costos de presentismo (70\%) a diferencia del ausentismo (30\%) (Collins et al. 2005). En Australia, el presentismo genera costos en la economía de $\$ 25.7$ billones de dólares por año. Sin embargo, el presentismo (0.89\%) a comparación del ausentismo $(0.47 \%)$, presenta mayores pérdidas en la productividad laboral de las empresas. Por otro lado, se pierden 2.1 días por trabajador, a causa del presentismo; mientras que se pierden 1.1 días por ausentismo (Medibank, 2008). Durante el 2008, un estudio realizado al Reino Unido para calcular los costos por enfermedad de la fuerza laboral, se encontraron pérdidas de 172 millones de días al año por ausentismo ( $£ 13$ billones de libras esterlinas); mientras que los costos por presentismo alcanzaron los $£ 15$ billones de libras esterlinas (Black, 2008). Un estudio realizado a 115 oficinistas, que trabajan a tiempo completo en un horario de 9 am a 5 pm, se ha encontrado que los trabajadores gastan diariamente un aproximado de 1 hora y 20 minutos en realizar actividades personales, lo cual genera una perdida en la productividad a causa del presentismo, de $\$ 8,875$ dólares anuales en promedio, por trabajador (D’Abate \& Eddy, 2007).

En Estados Unidos, se realizó un estudio que recopila información de diversas bases datos con la finalidad de medir las pérdidas de productividad en el trabajo o presentismo, en comparación a los costos por salud. Se encontró que los costos del presentismo son superiores a los costos médicos y representan el $61 \%$ de los costos asociados a la salud, como enfermedades mentales, migrañas, depresión, entre otros. (Goetzel, Long, Ozminkowski, Hawkins, Wang, \& Lynch, 2004). Además, se estimó que los costos del presentismo son superiores a los costos directos por salud, como migrañas o alergias (55\% a $82 \%$ ); mientras que en casos de hipertensión o cáncer $(6 \%$ a 53\%), los costos son inferiores (Schultz, Chin-Yu, \& Edington, 2009). Otro estudio realizado en los Estados Unidos a 9,282 trabajadores, entre el 2001 y 2003 basado en la encuesta National Comorbility Survey Replication, demuestra que el presentismo -a causa de la depresión - genera una pérdida anual de 18.2 días por trabajador, más del doble de días que el ausentismo (8.7 días). En términos monetarios, el presentismo genera costos de \$2,961 dólares por trabajador, cifra superior al ausentismo ( $\$ 1,420$ dólares). Proyectado a la fuerza laboral de los Estados Unidos, se pierden aproximadamente 51 millones de días equivalentes a $\$ 7,613$ millones de dólares por año; cifra superada por el presentismo, el cual genera pérdidas de 150.5 millones de días o $\$ 24,482$ millones de dólares por año. (Kessler et al, 2006a; Kessler et al, 2006b).

En el ámbito nacional, existen pocas investigaciones acerca del presentismo. Ruiz, Palomino, Zambrano, \& Llap (2006), realizaron un estudio que evalúa la productividad y los costos totales de un hospital durante el año 2003; se encontró que el 
$71 \%$ de la disminución de productividad es causado por el presentismo, mientras que el $29 \%$ a causa del tiempo perdido por ausentismo. Por otro lado, Vara (2012) ha estimado los costos del presentismo para las empresas, pero solo por consecuencia de la violencia contra las mujeres trabajadoras. Los costos ascienden en el Perú a 3 mil 500 millones de dólares, valor que contempla el presentismo producto de ser víctima de violencia familiar, ser agresor o ser testigo de violencia en los compañeros o compañeras de trabajo. Con relación a la proporción de costos frente al ausentismo y la rotación de personal, el presentismo representa el $73 \%$ del costo total para la empresa.

En efecto, el presentismo se convierte en un problema grave para el entorno empresarial, tanto para el empleador y empleado, pues hiere la productividad, la calidad de vida y la salud de los empleados (Saarvala, 2006), dicho problema es considerado como amenaza significativa a la eficiencia laboral y a la seguridad en el trabajo (Baker-McClearn, Greasley, Dale, \& Griffith, 2010). Los empleados que tienen presentismo no se encuentran totalmente concentrados en su trabajo, en lugar de ello, están más propensos a cometer errores, son menos productivos, brindan baja calidad en el servicio, se vuelven menos innovadores, todo ello tiene una gran repercusión dentro de la organización, tomando en cuenta que se es juzgado dentro de una empresa por los resultados obtenidos. Los errores accidentales por ausencias mentales pueden resultar extremadamente costosos (Gilbreath \& Karimi, 2012).

Se ha demostrado que el presentismo resulta más costoso y perjudicial para la productividad (Raju, 2012); sin embargo, en el Perú las empresas, aun no consideran al presentismo como uno de los costos más importantes en la gestión del recurso humano, ni siquiera han estimado sus costos e impacto en la productividad. Ante este vacío de informacion, la presente investigación busca cuantificar los costos del presentismo en las empresas del sector manufactura ubicadas en Lima.

\section{Objetivos}

1. Estimar y analizar los días laborales perdidos y los costos asociados a causa del presentismo laboral, tardanzas y ausentismos, medidos en valor agrega- do, de los trabajadores asalariados en las empresas manufactureras de Lima.

2. Estimar y analizar la diferencia de los costos a causa del presentismo laboral frente a los costos de ausentismo y tardanzas, de los trabajadores asalariados en las empresas manufactureras de Lima.

\section{Justificación}

Las empresas del sector manufacturera conocerán la situación actual de los costos del presentismo que afrontan, y podrán generar políticas empresariales enfocadas a la optimización de la productividad y disminución de los costos ocasionados por la pérdida de esta.

La investigación pretende eliminar paradigmas del sector empresarial que consideran al ausentismo, tardanzas y rotación de personal como los costos más altos de la productividad laboral. Asimismo, el estudio brinda un modelo que permite calcular los costos del presentismo, ausentismo y tardanzas; el cual puede ser reproducido por las empresas; para que puedan realizar estrategias que les permitan reducir estos costos.

\section{METODOLOGÍA}

\section{Diseño}

Descriptivo correlacional, utilizando la teoría contable del factor humano que mide los costos indirectos ocasionados por las lesiones mediante el valor del tiempo perdido por la reducción de la productividad o el ausentismo en el trabajo. La WHO (2007) recomienda determinar el periodo de evaluación de los costos anuales solo para el último año. Una forma de mejorar la competitividad de las empresas, es incrementando la productividad y el valor agregado.

\section{Población y muestra}

La población asciende a 35,556 empresas manufactureras de Lima, las cuales equivalen el $45.4 \%$ del total de empresas manufactureras a nivel nacional $(\mathrm{V}$ Censo Nacional Económico realizado por el INEI, 2008). Utilizando un muestreo para poblaciones infinitas, se calcula una muestra de 53 empresas manufactureras de Lima, las cuales tienen un total 
de 16,811 trabajadores conformado por 7,095 trabajadoras mujeres y 9,716 hombres. Las encuestas se aplican a un total de 903 trabajadores.

De las empresas que han aceptado participar en el estudio, el 79.2\% son grandes empresas (más de 100 trabajadores), el $7.55 \%$ son medianas empresas (51 y 100 trabajadores) y el $13.21 \%$ son pequeñas empresas ( 12 y 50 trabajadores).

\section{Instrumentos}

Se aplicaron cuestionarios estructurados, basado en el auto-reporte del desempeño de los trabajadores. El cuestionario está basado en el desarrollado por Vara (2012). Sin embargo, solo se han considerado las preguntas sobre información demográfica y laboral, salario hora, costos de presentismo, costos de ausentismo y costos de tardanza.

Utilizando el Análisis Factorial, Vara (2012) encontró que las escalas son independientes y válidas, saturando las variables en tres componentes diferenciados: En el primer componente satura el 100\% de los ítems de la escala de Presentismo (con pesos factoriales entre 0.35 y 0.77 ). En el segundo componente satura el 100\% de los ítems de la escala Tardanza (con pesos factoriales entre 0.56 y 0.83 ).
Finalmente, en el componente tres satura el 100\% de los ítems de la escala Ausentismo, con pesos factoriales fuertes $(0.54$ y 0.71$)$, evidenciando su validez. Respecto a la fiabilidad de las escalas, estas se muestran aceptables en todas las escalas.

Para el presente estudio, se calculó el nivel de fiabilidad por consistencia interna de las escalas de medición. Al respecto, los valores Alfa de Cronbach para las tres escalas (Presentismo $=0.772$, ausentismo $=0.639$, tardanzas $=0.713)$, las cuales tienen niveles adecuados, considerando la reducida cantidad de ítems para cada una de ellas. Las correlaciones media intra-clase son significativas y demuestran alta consistencia interna (entre 0.317 y 0.452 ).

En cuanto a la validez, el análisis de componentes principales de los ítems que componen las escalas, demuestran la validez de constructo de cada una de ellas. En el primer componente saturan los ítems de la escala de Presentismo, representando el 28.9\% de la varianza explicada de los datos. En el segundo componente saturan los ítems de la escala de tardanza, representando el 15.8\% de los datos; y en el componente 3 y 4, los ítems de la escala de ausentismo, representando el $18 \%$ de la varianza explicada.

Tabla No 01:

Número de empresas participantes de la muestra

\begin{tabular}{|l|r|r|}
\hline \multicolumn{1}{|c|}{ Sector } & Cantidad de empresas & (\%) \\
\hline Manufacturera - Industria alimenticia & 10 & 18.9 \\
\hline Manufacturera - Industria textil, cuero y calzado & 17 & 32.1 \\
\hline Manufacturera - Industria papel & 1 & 1.9 \\
\hline Manufacturera - Industria química & 5 & 9.4 \\
\hline Manufacturera - Industria metálica & 9 & 17 \\
\hline Manufacturera - Maquinaria y aparatos electrónicos & 4 & 7.5 \\
\hline Manufacturera - Minerales no metálicos & 1 & 1.9 \\
\hline Manufacturera - Caucho y plástico & 1 & 1.9 \\
\hline Manufacturera - Actividades de impresión & 5 & 9.4 \\
\hline & $\mathbf{5 3}$ & $\mathbf{1 0 0}$ \\
\hline
\end{tabular}

Fuente: Elaboración propia. 
Tabla No 02:

Estructura factorial de las escalas de presentismo, ausentismo y tardanza

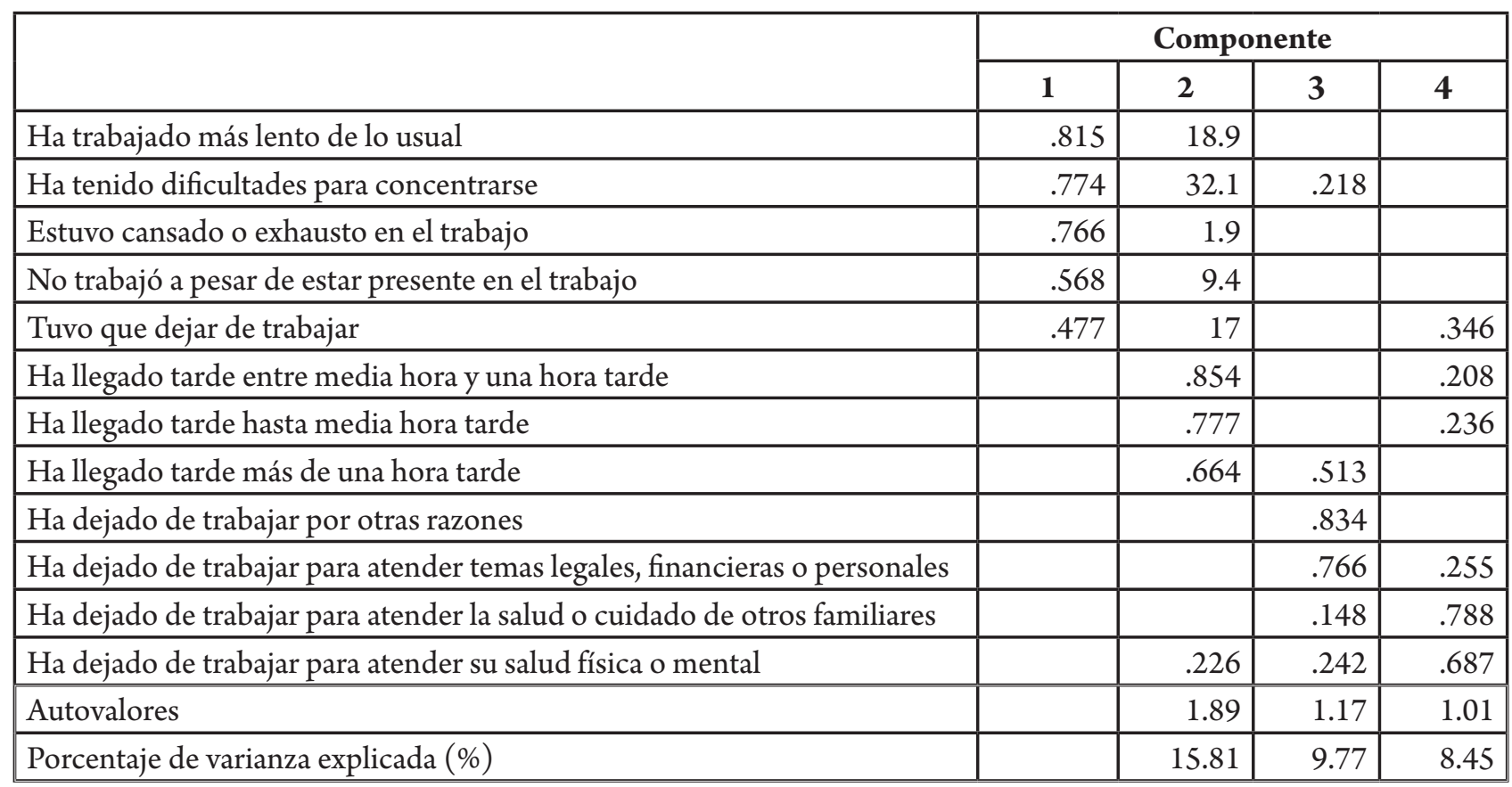

Método de extracción: Análisis de componentes principales. Método de rotación: Normalización Varimax con Kaiser. Determinante de la Matriz = 0.046; KMO = 0.765; Esfericidad de Bartlett $(\mathrm{X} 2=1599, \mathrm{gl}=66$, sig = 0.0001).

Fuente: Encuestas a trabajadores manufactureros de Lima.

\section{ANÁLISIS E INTERPRETACIÓN DE LA INFORMACIÓN}

Para el análisis de la información se utilizó el programa estadístico SPSS versión 19.

Estimación de días perdidos: Los días perdidos causados por presentismo, ausentismo y tardanza, fueron multiplicados por el total poblacional de hombres, mujeres, provenientes de la población económicamente activa adecuadamente empleada (con más de 35 horas de trabajo, contrato estable e ingresos por encima del promedio), solo de la población manufacturera de Lima.

Estimación de costos: Los impactos calculados en días perdidos de trabajo, son sumados conside- rando cada categoría de costos (ausentismo, presentismo, tardanza). Dado que el trabajador peruano promedio trabaja 288 días al año, perder esa cantidad de días es como perder la fuerza laboral del trabajador por un año. Se divide entonces la cantidad de días perdidos entre 288 días-trabajo, obteniendo la fuerza productiva perdida (en personas). El resultado es multiplicado por el valor nacional promedio de productividad laboral, el cual asciende a 72 mil soles año (en empresas manufactureras). El resultado es el valor agregado que las empresas pierden como producto de presentismo, ausentismo y tardanza. Los valores son convertidos a dólares americanos, asumiendo una tasa de cambio de 2.6 nuevos soles por dólar. 
Tabla No 03:

Valores nacionales considerados para las estimaciones de costos empresariales

\begin{tabular}{|l|r|}
\hline & \\
\hline PEAO remunerados (adecuadamente empleados) en Lima & $2,957,000$ \\
\hline PEAO manufacturera Lima & 328,472 \\
\hline Producto Bruto Interno (PBI, 2012) & USD\$ 182 410 millones \\
\hline Productividad laboral (Promedio nacional, 2011) manufactura & $1,206.25$ \\
\hline $\begin{array}{l}\text { Remuneración promedio mensual en nuevos soles (calculada en la } \\
\text { muestra) }\end{array}$ & 288 \\
\hline Días de trabajo anual por trabajador tiempo completo & USD\$ 2.6 \\
\hline Tasa de cambio dólares - soles & S \\
\hline
\end{tabular}

Fuente: INEI, ENAHO, ENDES, IV Censo Económico Nacional, BCRP.

\section{RESULTADOS Y DISCUSIÓN}

\section{Resultados}

Participaron 903 trabajadores y trabajadoras, siendo el $36.7 \%$ varones y el $63.3 \%$ mujeres. La edad promedio de los trabajadores es de 32.31 años (D.E. $=9,85)$. El promedio de horas de trabajo es de 49.24 horas semanales (D.E. $=8.48$ ), con un sueldo mensual promedio de 1,206.25 nuevos soles (D.E. $=1,198.1$ ), con pagos que oscilan entre 338 nuevos soles hasta los 15 mil nuevos soles.

En cuanto al ausentismo, el $40.8 \%$ reporta algún tiempo de ausentismo en el último mes para atender su salud física o mental. Otro $37.3 \%$ se han ausentado para atender la salud o cuidado de sus familiares. El $25.2 \%$ se ha ausentado para atender temas legales, financieros o personales; y, finalmente, el $23.6 \%$ se ha ausentado por otras razones. Se observa que las principales razones de ausentismo son las ligadas a la salud personal o familiar.

En cuanto a la tardanza, estas son poco frecuentes. Las tardanzas más prevalentes son aquellas menores a media hora (32.5\%), que las de una hora (11.6\%) o más de una hora (5.5\%). Esto puede ocurrir porque en la mayoría de empresas, las tardanzas tienen un tiempo límite y luego pasan a ser consideradas ausentismo.

En cuanto al presentismo, el $46.5 \%$ de trabajadores ha tenido dificultades para concentrarse laboral- mente, al menos un cuarto del tiempo en las últimas cuatro semanas. Por otro lado, el $44.2 \%$ afirma haber trabajo más lento de lo usual en las últimas cuatro semanas. Los porcentajes son mayores en cuanto a sentirse cansado o exhausto, donde el 50.2\% afirma haber estado cansado o exhausto en el trabajo en el último mes, al menos un cuarto del tiempo. En cuanto a la prevalencia de cero productividad, el $12.8 \%$ de trabajadores afirma que en las últimas cuatro semanas no trabajó a pesar de estar presente en el trabajo. De igual forma, el $7.1 \%$ tuvo que dejar de trabajar por diversas razones en al menos un cuarto del tiempo de su jornada.

En términos globales, se observa que la prevalencia de tardanza es de $22.1 \%$, para el ausentismo es de $42.4 \%$ y para el presentismo es $58.5 \%$ (ver Figura $\mathrm{N}^{\circ} 01$ ).

El costo promedio anual del presentismo es muy superior al costo de ausentismos y tardanzas (Ver Tabla $\mathrm{N}^{\circ}$ 04). Mientras que el costo promedio de tardanzas es de 143.6 nuevos soles anuales, el costo de ausentismo es diez veces superior (1,697 nuevos soles) y, del presentismo, es treinta veces más alto (3,897 nuevos soles). Las tardanzas y el ausentismo suele descontarse del sueldo del trabajador, pero no ocurre así con el presentismo. Estos valores no contemplan el valor agregado, solo están basados en función del salario. 
Figura $\mathbf{N}^{\circ}$ 01: Prevalencia conjunta de tardanza, ausentismo y presentismo en empresas manufactureras de Lima

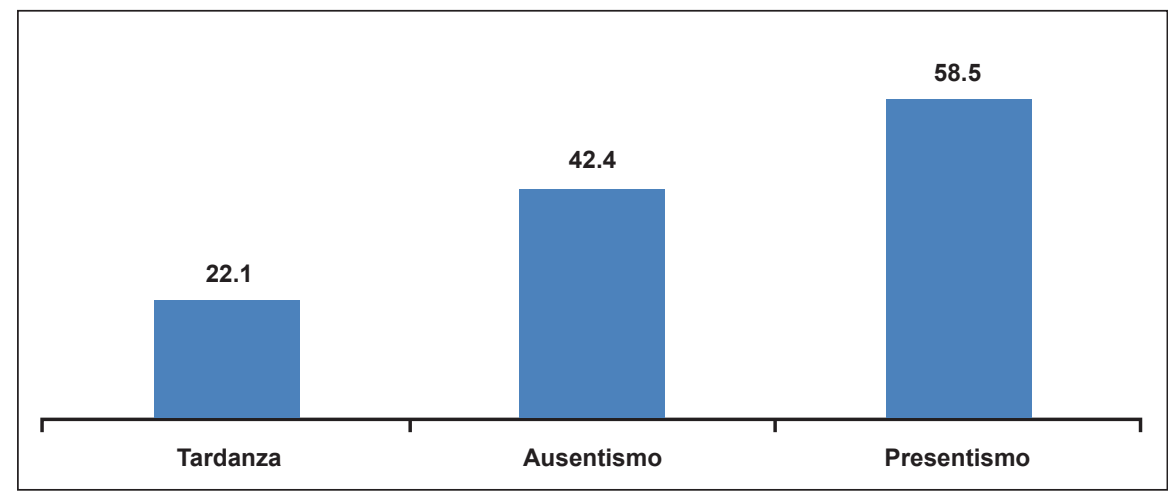

* Se considera solo aquellos casos donde se irrogó algún costo oculto.

Fuente: Encuestas a trabajadores manufactureros de Lima. Elaboración propia.

Tabla No 04:

Costo promedio anual por trabajador (expresado en nuevos soles), en función del ausentismo, presentismo y tardanza (sin considerar valor agregado)

\begin{tabular}{|l|c|c|c|}
\hline & Presentismo & Tardanza & Ausentismo \\
\hline N incluida & 484 & 172 & 337 \\
\hline N excluida ${ }^{*}$ & 419 & 731 & 566 \\
\hline Media & $3,897.7$ & 143.6 & $1,697.7$ \\
\hline Desv. Estándar & $6,312.4$ & 225 & $2,563.4$ \\
\hline
\end{tabular}

* No presentaron tardanza, ausentismo o presentismo.

Fuente: Encuestas a trabajadores manufactureros de Lima. Elaboración propia

En la Tabla $N^{\circ} 5$ se presentan los costos ocultos de presentismo, tardanzas y ausentismo en función del valor agregado perdido por año en las empresas manufactureras de Lima. Para ser conservadores, en estos cálculos solo se han considerado a la población económicamente activa adecuadamente empleada (con contrato mínimo de 35 horas semanales y beneficios sociales, y con ingresos mayores al mínimo referencial), la cual asciende en Lima a casi tres millones de trabajadores (Encuesta Permanente de Empleo del INEI, 2013).

Tabla No 05:

Costos ocultos de presentismo, tardanzas y ausentismo en función del valor agregado perdido por año en las empresas manufactureras de Lima (expresado en nuevos soles)

\begin{tabular}{|l|c|c|c|c|}
\hline & Prevalencia (N) & $\begin{array}{c}\text { Costo anual en salarios } \\
(\mathbf{S} / .)\end{array}$ & $\begin{array}{c}\text { Fuerza laboral } \\
\text { perdida }\end{array}$ & $\begin{array}{c}\text { Valor agregado } \\
\text { perdido (S/.) }\end{array}$ \\
\hline Presentismo & 192,156 & $748,973,346.2$ & 51,743 & $3,725,463,276$ \\
\hline Tardanza & 72,592 & $10,424,197.93$ & 720 & $51,850,932.71$ \\
\hline Ausentismo & 139,272 & $236,446,859.8$ & 16,335 & $1,176,108,733$ \\
\hline Total & 404,020 & $995,844,403.9$ & 68,798 & $4,953,422,942$ \\
\hline
\end{tabular}

Notas: PEAO adecuadamente empleada en Lima para el sector manufacturero $=328,472$ trabajadores; Remuneración promedio mensual (calculada en la muestra $=1,206.25$ ).

Fuente: Encuestas a trabajadores manufactureros de Lima. Elaboración propia. 
Se observa que el costo anual considerando salarios en las tardanzas y ausentismo es de 246 millones 871 nuevos soles aproximadamente, los cuales suelen ser descontados de la remuneración del personal. Pero este valor no es lo la pérdida en valor agregado, solo representan lo descontado. En realidad, la pérdida en valor agregado por tardanzas y ausentismo asciende a $1 \mathrm{mil} 227$ millones $960 \mathrm{mil}$ nuevos soles; cerca de un billón de nuevos soles que no son recuperados de manera alguna. En el caso del presentismo, el panorama es más preocupante, pues el costo por este concepto no es descontado al no tener forma de identificarlo efectivamente. Son 3 mil 725 millones 463 mil nuevos soles perdidos por año a causa del presentismo en Lima.

Considerando solo los costos en valor agregado, el presentismo representa el $75.2 \%$ de los costos, frente al $23.7 \%$ de los costos provenientes del ausentismo y el $1.1 \%$ de las tardanzas.

Figura $\mathrm{N}^{\circ}$ 02: Distribución porcentual del costo total en valor agregado, en función del ausentismo, presentismo y tardanza

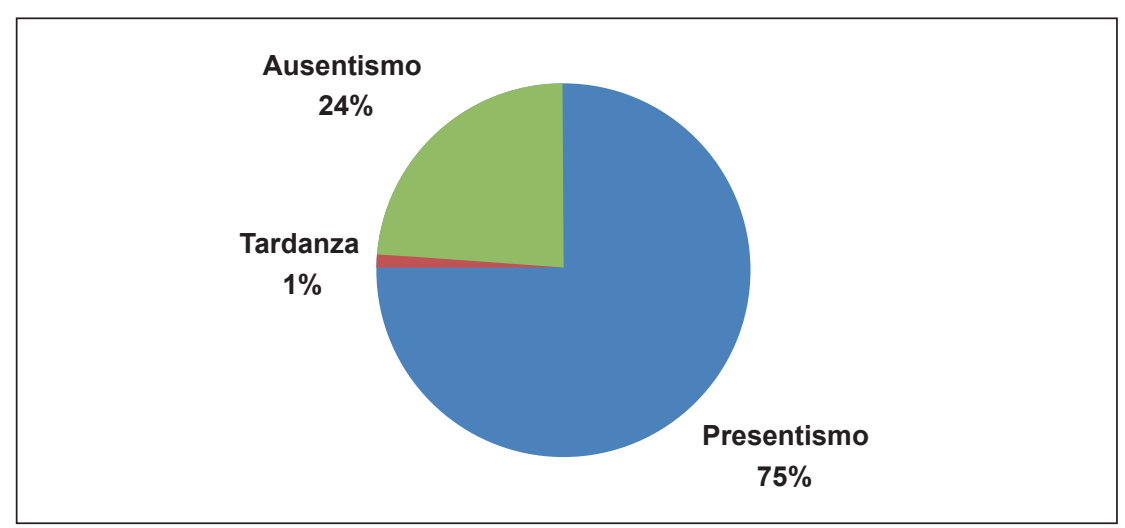

Fuente: Encuestas a trabajadores manufactureros de Lima. Elaboración propia.

\section{Discusión}

El presentismo es un problema perjudicial para las personas y empresas, que puede empeorar si es que no se toman las medidas para lidiar contra él. Existen diferentes causas para el presentismo, pero si se toma las medidas adecuadas los síntomas pueden disminuir (Saarvala, 2006). En efecto, las condiciones de salud negativas reducen significativamente la productividad laboral, por eso la evaluación del presentismo es una oportunidad para que los empleadores mejoren el rendimiento de sus empleados, a través de inversiones estratégicas en el cuidado de la salud y en un ambiente laboral saludable (Medibank, Sick at Work: The cost of presenteeism to your business and the economy, 2011; Shepellfgi, 2007; Saarvala, 2006).

En la presente investigación se ha encontrado que los costos del presentismo representan, en relación al ausentismo y tardanza, el $75.2 \%$; es decir, tres cuar- tos de las pérdidas en productividad laboral. Estos valores son coherentes con los obtenidos en estudios previos, realizados en países de altos ingresos como Estados Unidos, Australia y Reino Unido. Collins et al (2005) encuentra que el presentismo en Estados Unidos representa el $70 \%$ de las pérdidas en desempeño, a diferencia del ausentismo (30\%). De igual forma, Goetzel et al (2004) encontró que el presentismo representa el $61 \%$ de los costos asociados a la salud en las empresas; y Saarvala (2006) encontró que el presentismo representa el $60 \%$ del costo total por enfermedad de un trabajador. En el Perú, Vara (2012) encontró que las empresas peruanas pierden productividad como consecuencia de la violencia intrafamiliar de sus trabajadores, y el presentismo representa el $73 \%$ del costo total, en relación al ausentismo, rotación y tardanza de personal. Otro estudio congruente con estos resultados es el realizado en el Perú por Ruíz et al (2006) donde encontraron que los costos hospitalarios por pérdida de productividad 
eran del $71 \%$ para el caso del presentismo y del 29\% para el ausentismo.

El presentismo trae enormes pérdidas invisibles para las empresas. En la presente investigación se ha encontrado que el presentismo representa una pérdida de 3.7 billones de nuevos soles por año para Lima, un equivalente a 1.4 billones de dólares americanos por año. Estos valores, solo se corresponden al sector manufacturero de Lima; no a todos los sectores empresariales ni en todo el territorio nacional. Otros estudios internacionales estiman costos de presentismo a nivel país y de todos los sectores empresariales, encontrando por ejemplo que en Estados Unidos las empresas pierden entre 150 y 250 billones de dólares al año (Hemp, 2004); o en Australia, cuya pérdida por presentismo asciende a 25.7 billones de dólares (Medibank, 2008); o en Reino Unido, cuyas pérdidas ascienden a 15 billones de libras esterlinas por año (Black, 2008). En todos los casos, las pérdidas son muy superiores a las del ausentismo, tardanza o rotación de personal.

Estos resultados, coherentes con los encontrados en los antecedentes, evidencian la imperiosa necesidad de gestionar el presentismo; que desde las gerencias de recursos humanos, de personal o equivalentes, se debe prestar tanta atención como la que se dirige a otras áreas de desempeño.

El presentismo por sí solo no va a desparecer, los empleadores son quienes tienen que tomar medidas para hacerle frente a la situación con la finalidad de crear ambientes saludables y productivos para sus empleados, de esta forma, lograr mayor rentabilidad (Medibank, Sick at Work: The cost of presenteeism to your business and the economy, 2011; Shepellfgi, 2007; Levy, 2003; Klachefsky, 2012; COMCARE, 2012).

\section{CONCLUSIONES}

1. Los costos del presentismo laboral representan una pérdida significativa para el sector de manufactura en Lima. El presentismo representa una pérdida de 3 mil 725 millones 463 mil nuevos soles por año para Lima, un equivalente a 1,432 millones 870 mil dólares americanos por año, o al $0.78 \%$ del Producto Bruto Interno.
2. El presentismo laboral, las tardanzas y los ausentismos representan un número significativo de días perdidos, en las empresas manufactureras de Lima. Se pierden 19 millones 813 mil días de trabajo, un equivalente a 68 mil 798 trabajadores tiempo completo por año. De estos días perdidos, 207 mil 403 días corresponden a tardanzas, 4 millones 704 mil 434 a ausentismos y 14 millones 901 mil 853 días a presentismo.

3. Los costos del presentismo laboral, tardanzas y ausentismos para el sector de manufactura, representan una pérdida significativa en la productividad laboral, medido en valor agregado. Las pérdidas en valor agregado de estos tres conceptos es de 4,953 billones 423 mil nuevos soles aproximadamente, un equivalente a 1,905 millones de dólares americanos.

4. Los costos del presentismo, para el sector de manufactura de Lima, representan un alto porcentaje de los costos totales, en comparación de los costos por ausentismo y tardanzas. Los costos de presentismo representan el $75.2 \%$ de los costos en valor agregado, en comparación con los costos de tardanzas (1.04\%) y ausentismos (23.74\%).

\section{RECOMENDACIONES}

1. Los costos del presentismo, para el sector de manufactura, representan un costo mucho mayor, en comparación a los costos por ausentismo y tardanzas. Sin embargo, las empresas no cuentan con un sistema de gestión para controlarlo o prevenirlo. El presentismo es la principal causa de errores y accidentes laborales y, su prevención oportuna, redunda en la reducción de costos indirectos y de siniestralidad. Por tanto, se recomienda crear modelos de gestión del desempeño que incluya dentro de sus componentes, el control y manejo del presentismo, enfatizando en la prevención.

2. El presentismo ocurre por diversas causas y básicamente se manifiesta en distracción laboral y agotamiento físico y mental; pero aún se desconocen las principales variables que aumentan la probabilidad de experimentar presentismo, pues las investigaciones en el campo aún son iniciales. De lo dicho, se recomienda realizar futuras inves- 
tigaciones para identificar los factores que aumentan la distracción laboral, principalmente aquellos ligados al conflicto familia-trabajo; así como a los factores que condicionan el agotamiento físico y mental, como los hábitos alimenticios y de sueño.

3. En la presente investigación se ha estimado los costos del presentismo en la industria manufacturera, pero se desconoce los efectos en las empresas de servicios, por lo que se recomienda replicar la investigación en este tipo de empresas.

\section{REFERENCIAS BIBLIOGRÁFICAS}

1. ARONSSON, G., \& GUSTAFSSON, K. (2005) Vacation-still an issue of workers' protection? An empirical study of vacation and recuperation. International Journal of Health Services, 35(1), 143-169.

2. BAKER-McClearn, D., GREASLEY, K., DALE, J., \& GRIFFITH, F. (2010) Absence management and presenteeism: the pressures on employees to attend work and the impact of attendance on performance. Human Resource Management Journal, 20(3), 311-328.

3. BLACK, D. (2008) Working for a healthier tomorrow. Londres: The Cross-government Health, Work and Well-being Programme.

4. COLLINS, J., BAASE, C., SHARDA, C., OZMINKOWSKI, R., NICHOLSON, S., BILLOTI, S., BERGER, M. (2005) The Assessment of Chronic Health Conditions on Work Performance, Absence, and Total Economic Impact for Employers. Journal of Occupational and Environmental Medicine, 47, 547-557.

5. COMCARE. (2012) Benefits to Business: The evidence for investing in worker health and wellbeing. COMCARE. Putting you first, 1-6.

6. D'ABATE, C., \& Eddy, E. (2007) Engaging in Personal Business on the job: Extending the Presenteeism Construct. Human Resource Development Quarterly, 18(3), 361-383.

7. FERNÁNDEZ, E., AVELLA, L., \& FERNÁNDEZ, M. (2003) Estrategia de producción. McGraw Hill: Madrid.
8. GARCÍA, M. (2011) Problemática multifactorial del absentismo laboral, el presentismo y la procrastinación en las estructuras en que se desenvuelve el trabajador. Medicina y seguridad del trabajo, 57(223), 111-120.

9. GILBREATH, B., \& KARIMI, L. (2012) Supervisor Behavior and Employee Presenteeism. International Journal of Leadership Studies, 7(1), 114-131.

10. GOETZEL, R.,LONG, S., OZMINKOWSKI, R., HAWKINS, K., WANG, S., \& LYNCH, W. (2004) Health, Absence, Disability, and Presenteeism Cost Estimates of Certain Physical and Mental Health Conditions Affecting U.S. Employers. Journal Occupational Environment, 398-412.

11. HEMP, P. (2004). Presenteeism: At Work- But out of It. Hardvard Business Review, 82, 49-58.

12. JOHNS, G. (2010). Presenteeism in the workplace: A review and research agenda. Journal of Organizational Behavior (31), 519-542.

13. KESSLER, R., AKISKAL, H., \& AMES, M. (2006). Prevalence and Effects ofmood disorders on work performance in a nationally representative sample. American Journal of Psychiatry, 163(9), 1561-1568.

14. KESSLER, R., AKISKAL, H., AMES, M., BIRNBAUM, H., GREEMBERG, P., HIRSCHFELD, R., WANG, P. (2006) Prevalence and Effects of Mood Disorders on Work Performance in a Nationally Representative Sample of U.S. Workers. American Journal of Psychiatry, 163(9), 15611568.

15. LEVY, D. (Enero de 2003). A Method for Assessing the Extent of Family Caregivers in the Workplace and their Financial Impact. American Association for Caregiver Education, 1-19.

16. MEDIBANK. (2008). The cost of workplace stress in Australia. Melbourne: Medibank Private Limited.

17. MEDIBANK. (2011). Sick at Work: The cost of presenteeism to your business and the economy. Part of the Medibank research seriesSick at Work, 12. 
18. RAJU, P. G. (2012) Different Manifestations of Presenteeism at Workplace: A Study of Behavior of Employees in I.T. Sector in Indi. Obtenido de http://ssrn.com/abstract=2039848 or http:// dx.doi.org/10.2139/ssrn.2039848

19. RUIZ, F., PALOMINO, J., ZAMBRANO, R., \& Llap, C. (2006) Prevalencia, impacto en la productividad y costos totales de las principales enfermedades en los trabajadores de un hospital al sur del Perú en el año 2003. Revista Médica Heredia, 17(1), 28-34.

20. SAARVALA, E. (2006) Presenteeism: The latest attack on economic and human productivity.
Human Resources Management - University of Toronto.

21. SCHULTZ, A., Chin-Yu, C., \& Edington, D. (2009). The Cost and Impact of Health Conditions on Presenteeism to Employers. Pharmacoeconomics, 27(5), 365-378.

22. SHEPELLFGI (2007) Getting Employees Back In The Game. Healthy working: A workplace wellness update, 1-2.

23. VARA, A. (2012) Los costos empresariales de la violencia contra las mujeres en el Perú. Lima: Universidad de San Martín de Porres, GIZ, BMZ, Programa ComVoMujer. 